\title{
Phase matching strategy for the undulator system in the European X-ray Free Electron Laser
}

\author{
Yuhui Li and Joachim Pflueger \\ The European XFEL GmbH, Notkestrasse 85, 22607 Hamburg, Germany
}

(Received 20 May 2016; published 6 February 2017)

\begin{abstract}
The undulator system in the European X-ray Free Electron Laser is mainly comprised of 5-m long undulator segments and $1.1 \mathrm{~m}$ long intersections in between. The longitudinal component of the electrons' velocity is reduced when traveling inside an undulator due to the wiggle motion. Therefore the optical phase is detuned. The detune effect is also from the undulator fringe field where electron longitudinal speed also deviates from the oscillation condition. The total detune effect is compensated by a magnetic device called phase shifter, which is correspondingly set for a specific undulator gap. In this paper we investigate the homogeneity of the fringe field from different undulators. Different phase matching criteria are studied. The field fitting technique for the phase matching in high accuracy is demonstrated in detail. The impact by air coil is also studied. Eventually the matching test by spontaneous radiation simulation is made. A test method for high sensitivity to matching error is proposed.
\end{abstract}

DOI: 10.1103/PhysRevAccelBeams.20.020702

\section{INTRODUCTION}

High gain free electron lasers (FELs) using the principle of self-amplified spontaneous emission (SASE) are so far the only way to generate FEL radiation in the hard $\mathrm{x}$-ray range [1-5]. For vacuum ultraviolet radiation, alternatives, such as harmonic generation, exist that generate more stable radiation [6]. However, aside from such differences both require long undulator systems with lengths from tens of meters up to about 200 meters depending on the radiation wavelength and electron beam parameters. Such a long undulator system cannot be built as a continuous device. For practical reasons of manufacturing it must be segmented into lengths around typically $5 \mathrm{~m}$ maximum.

In the intersection between two undulator segments auxiliary equipment such as quadrupoles, beam position monitors, vacuum pumps, correctors, etc. can be installed. For the FEL process the interruption of the undulator implies a problem: The longitudinal speed of the electrons in the intersection is different from that inside the undulator and therefore the optical phase matching between laser field and electron motion is perturbed.

In a fixed gap undulator system such as FLASH $[1,2]$ or LCLS I [3] phase matching can be obtained by choosing the intersection length and tuning the end fields of the undulator segments properly. The phase matching in a gap tunable system is more complicated since the phase mismatch in the intersection changes with undulator gap. With a phase shifter, a small magnetic chicane, an

Published by the American Physical Society under the terms of the Creative Commons Attribution 3.0 License. Further distribution of this work must maintain attribution to the author(s) and the published article's title, journal citation, and DOI. additional delay is induced in the electron orbit. By properly selecting the phase shifter strength and hence the delay the optical phase can be matched at any gap [7].

The European X-ray Free Electron Laser (EXFEL) facility is currently under construction [5]. It is a large project driven by a 1.8 kilometer long superconducting linac. The SASE FEL is used throughout the European XFEL. An electron beam is accelerated up to a maximum of $17.5 \mathrm{GeV}$. Then it is guided through the undulator system to generate high quality soft and hard $\mathrm{x}$ rays. The radiation wavelength can be changed by variation of the undulator gap and in addition the beam energy: The hard $\mathrm{x}$-ray range from 0.1 to $0.4 \mathrm{~nm}$ is covered by two undulator lines called SASE1 and SASE2. The soft $\mathrm{X}$-ray wavelength range from 0.4 to $1.6 \mathrm{~nm}$ is covered by one undulator line called SASE3.

For the hard x-ray FELs SASE1 and SASE2 two undulator systems with a total length of about $210 \mathrm{~m}$ each comprising 35 U40 type undulator segments are needed to reach saturation. For the soft x-ray FEL SASE3 the undulator system is comprised of 21 U68 type undulator segments with a total length of about $112 \mathrm{~m}$.

Both U40 and U68 use the same standard $5 \mathrm{~m}$ long mechanical support structure. The intersections for all SASE beam lines are standardized as well. They are $1.1 \mathrm{~m}$ long. All use the same type of permanent phase shifter. In total 91 undulator segments are built and therefore 88 phase shifters are needed.

Undulators and phase shifters are well characterized before installation: Undulators were accurately tuned for small trajectory and phase errors in the operational gap range $[8,9]$. After tuning the characterization for each segment includes high resolution field maps as a function of gap in $0.5 \mathrm{~mm}$ steps. They are the basis for the calculation of device properties such as K-parameter, 
optical phase errors, trajectory wander, etc. All phase shifters were well tuned and characterized as well [10-13]. Field integral errors were corrected below XFEL tolerance levels for all gaps [12]. The phase integral characterizing the strength of a phase shifter was systematically recorded for all phase shifter gaps. All data are stored in the undulator system database (USDB).

An undulator system comprised of many segments is best controlled by the K-parameter, which directly relates to the radiation wavelength, and not by the individual gaps, which differ slightly from segment to segment due to manufacturing and material tolerances. The K-parameter plays a key role and needs to be provided with an accuracy of $\Delta K / K \leq 2 \times 10^{-4}$. The $\mathrm{K}$ (gap) or the inverted the Gap $(\mathrm{K})$ relation is available for each undulator segment and stored on the USDB with high precision.

This paper concentrates on the phase matching of undulator segments and the generation of the lookup tables for the phase shifters using the data stored in the USDB. The relevant theory is derived. These lookup tables are needed for the commissioning of the undulator system. For commissioning the K-parameter needs to be provided with the accuracy given above. It has been demonstrated that the magnetic data are accurate enough [14].

\section{PHASE MATCHING STRATEGY}

\section{A. Practical situation}

In order not to deteriorate the FEL process the $\mathrm{K}$-parameters of the individual undulator segments need to be provided with an accuracy $\Delta K / K \leq \rho$, where $\rho$ is the Pierce parameter, which for EXFEL is about $2 \times 10^{-4}$. Therefore, in an undulator system the gaps of individual undulator segments need to be adjusted to match very specific $\mathrm{K}$ values that are required to make all undulator segments resonant to the same $\mathrm{x}$-ray wavelength in the presence of energy losses.

On the other hand, undulator field measurements are made by controlling the gap mechanically with micrometer precision. Therefore, the K-parameters for each segment need to be evaluated by interpolation from measurements made at different gaps.

\section{B. Phase matching criteria}

Figure 1 illustrates two undulator cells. Each cell is subdivided into four regions: Going from left to right the beginning of a cell is chosen in the field-free region in front of the undulator, right after the end of the upstream phase shifter. The region from the beginning of the cell to the beginning of undulator bulk poles, including the drift space and the undulator fringe field, is called entrance fringe. The phase advance in this part is $\varphi_{\text {Entr }}$. The periodic field region inside the undulator is called bulk field. The phase advance over this region is $\varphi_{\text {Bulk }}$. Ideally at the first harmonic the phase advance in this region is $2 \pi$ per period. Similar to the entrance fringe the region from the end of the bulk field to the beginning of the phase shifter is called exit fringe with the phase advance $\varphi_{\text {Exit }}$. In the field free region after the exit fringe the phase shifter is placed. The phase advance over the phase shifter is $\varphi_{\mathrm{PS}}$. Since the phase shifter has very low fringe fields [7], it does not interfere with the undulator and the spatial extension of its fringe field is very close to its short physical length of only $230 \mathrm{~mm}$. The region after the phase shifter is again field free. Accordingly the phase advance over the first undulator cell can be written as $\varphi_{\text {Und }, 1}=\varphi_{\text {Entr }, 1}+\varphi_{\text {Bulk }, 1}+\varphi_{\text {Exit }, 1}+\varphi_{\mathrm{PS}, 1}$.

Only planar undulators are used at the European XFEL, so only the vertical field $\mathrm{B}_{y}$ plays a role. The horizontal field component $\mathrm{B}_{x}$ resulting from field errors is less than $\approx 10^{-3}$ times smaller than the main field component and can be neglected. The spontaneous energy radiated per unit solid angle per unit frequency interval $d I(\omega) / d \Omega$ is $d I(\omega) / d \Omega=2|A(\omega)|^{2}[15,16]$. The on-axis radiation of a planar undulator $A(\omega)$ is written as

$A(\omega)=i\left(\frac{e^{2}}{8 \pi^{2} c}\right)^{1 / 2} \frac{k}{\gamma} \int_{0}^{z} I_{1 y}\left(z_{1}\right) e^{i k\left[\int_{0}^{z_{1}} \frac{1}{2 \gamma^{2}}\left[1+I_{1 y}^{2}\left(z_{2}\right) d z_{2}\right]\right]} d z_{1}$,

where $k=\frac{2 \pi}{\lambda_{\text {Rad }}}$ is the wave number, $\lambda_{\text {Rad }}$ the radiation wavelength, $c$ is the speed of light, $e$ the electron charge and $\gamma$ the kinetic energy in units of the electron rest mass. $I_{1 y}$ is proportional to the first field integral: $I_{1 y}=\frac{e}{m c} \int_{0}^{z} B_{y}\left(z^{\prime}\right) d z^{\prime}$. The argument of the exponential function in Eq. (1) is the optical phase function [16]:

$$
\varphi\left(z_{1}\right)=\int_{0}^{z_{1}} \frac{k}{2 \gamma^{2}}\left[1+I_{1 y}^{2}\left(z_{2}\right) d z_{2}\right]
$$

For convenience a normalized form of $A, A_{n}$, is used. In terms of the optical phase $\varphi\left(z_{1}\right)$ and the first field integral Eq. (1) is rewritten

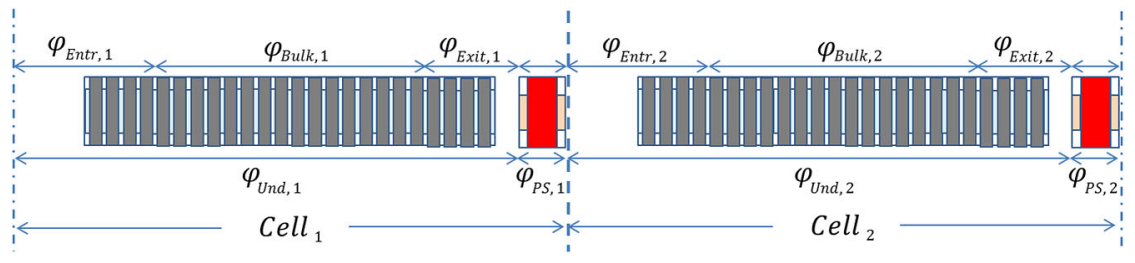

FIG. 1. Definition of the different field regions in two sample undulator cells. 


$$
A_{n}=\int_{0}^{z} I_{1 y}\left(z_{1}\right) e^{i \varphi\left(z_{1}\right)} d z_{1}
$$

$A_{n}$ is complex. $A_{n}$ for two undulator segments is the vector sum of two complex numbers. Using the normalized form is written as

$$
A_{n, \text { sum }}=A_{n, 1}+e^{i\left(\varphi_{\mathrm{Und}, 1+} \varphi_{\mathrm{PS}}\right)} A_{n, 2},
$$

where $A_{n, 1}$ and $A_{n, 2}$ denote $A_{n}$ of the first and the second undulator, respectively. Both undulators and the corresponding radiation are similar but not identical. Each can be expressed in complex polar coordinates as

$$
A_{n}=\left|A_{n}\right| e^{i \psi} .
$$

$\psi$ is the phase of the radiation complex amplitude $A_{n}$ and must not be confused with the optical phase $\varphi$.

The total $A_{n}$ of two undulators is the complex sum of two. Equation (4) is then rewritten as

$$
A_{n, \mathrm{sum}}=\left|A_{n, 1}\right| e^{i \psi_{1}}+\left|A_{n, 2}\right| e^{i \psi_{2}} \cdot e^{i\left(\varphi_{\mathrm{Und}, 1}+\varphi_{\mathrm{PS}}\right)} .
$$

The maximum for $\left|A_{n, \text { sum }}\right|$ is obtained if the condition

$$
\psi_{1}=\varphi_{\mathrm{und}, 1}+\varphi_{\mathrm{PS}}+\psi_{2}+2 n \pi
$$

is fulfilled where $n$ is an integer. Equation (7) is the criterion for calculating the phase matching. Figure 2 gives an illustrative description. $A_{n}$ of the two undulators are plotted in the complex plane. If Eq. (7) is fulfilled the resulting $A_{n}$ is longest if $A_{n, 1}$ and $A_{n, 2}$ are collinear.

In order to explain the relation between the phase of the complex $A_{n}, \psi$, and the optical phase $\varphi$, we analyze an undulator field comprised by an ideal bulk field and two ending fields. Equation (3) is expressed by the integral over different parts of an undulator as sketched in Fig. 1:

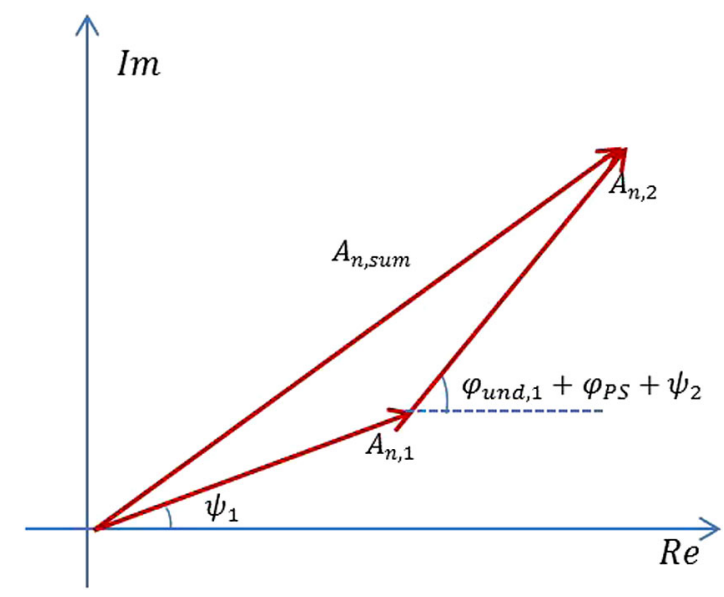

FIG. 2. Sum of the $A_{n}$ of two undulators. The length of $A_{n}$ is maximum if the condition $\psi_{1}=\varphi_{\text {und, } 1}+\varphi_{\mathrm{PS}}+\psi_{2}+2 n \pi$ is satisfied.
$A_{n}=\delta_{\text {entr }}+e^{i \varphi_{\text {Entr }}} \int_{L_{\text {entr }}}^{L_{\text {entr }}+L_{\text {bulk }}} I_{1 y}\left(z_{1}\right) e^{i \varphi\left(z_{1}\right)} d z_{1}+\delta_{\text {exit }}$,

where $\delta_{\text {entr }}=\int_{0}^{L_{\text {entr }}} I_{1 y}\left(z_{1}\right) e^{i \varphi\left(z_{1}\right)} d z_{1}, \delta_{\text {exit }}=e^{i\left(\varphi_{\text {Entr }}+\varphi_{\text {bulk }}\right)} \times$ $\int_{L_{\text {entr }}+L_{\text {bulk }}}^{L_{\text {ent }}+L_{\text {exit }}} I_{1 y}\left(z_{1}\right) e^{i \varphi\left(z_{1}\right)} d z_{1}$ are the contributions to $A_{n}$ from the undulator end fields at the entrance and exit. In the end field sections the poles have different strength from the bulk and the optical field is out of resonance. $\delta_{\text {entr }}$ and $\delta_{\text {exit }}$ only contribute to the optical phase advance $\varphi_{\text {Entr }}$ and $\varphi_{\text {Exit }}$ but at resonance their contributions to the modulus of $A_{n}$ are negligibly small. Therefore Eq. (8) is simplified:

$$
A_{n} \approx e^{i \varphi_{\text {Entr }}} \int_{L_{\text {entr }}}^{L_{\text {entr }}+L_{\text {bulk }}} I_{1 y}\left(z_{1}\right) e^{i \varphi\left(z_{1}\right)} d z_{1} .
$$

The bulk field is expressed by sinus function and expressed in terms of Bessel functions [17]:

$A_{n} \approx-e^{i \varphi_{\text {Entr }}} K \cdot N \frac{\lambda_{u}}{2}\left[J_{0}\left(\frac{K^{2}}{1+0.5 K^{2}}\right)-J_{1}\left(\frac{K^{2}}{1+0.5 K^{2}}\right)\right]$,

where $N$ is the number of periods. $J_{0}$ and $J_{1}$ are the Bessel functions of integer order 0 and 1.

Equation (10) shows that the contribution from the bulk field to the vector potential is real, since the phase advance per period is $2 \pi$. Therefore $\varphi_{\text {bulk }}=0$ and has no effect on the phase of $A_{s}$. So $\psi$ is determined by the entrance field of an undulator:

$$
\psi=\varphi_{\text {entr }} .
$$

Because in an ideal undulator $\varphi_{\text {bulk }}=0$ and $\varphi_{\text {und }}=$ $\varphi_{\text {entr }}+\varphi_{\text {bulk }}+\varphi_{\text {exit }}$, the criteria of Eq. (7) for phase matching can be written in the format of optical phase:

$$
\varphi_{\mathrm{exit}, 1}+\varphi_{\mathrm{PS}}+\varphi_{\mathrm{entr}, 2}=2 n \pi .
$$

Equation (12) gives a criterion for the phase matching of two undulators: The optical phase from the last bulk pole of the upstream undulator should change integral times of $2 \pi$ to the first bulk pole of the next undulator. However, Eq. (12) assumes an ideal bulk field of the undulator with results in a phase advance of $2 \pi$ per period over the whole undulator. In practice phase errors are unavoidable and the accuracy of Eq. (12) is affected by field errors.

\section{MATCHING OF UNDULATORS}

\section{A. Investigation of simple matching techniques}

The method described in the previous section needs field maps at a specific K-parameter. This requires an appropriate interpolation scheme to deduce these data from adjacent gaps. 

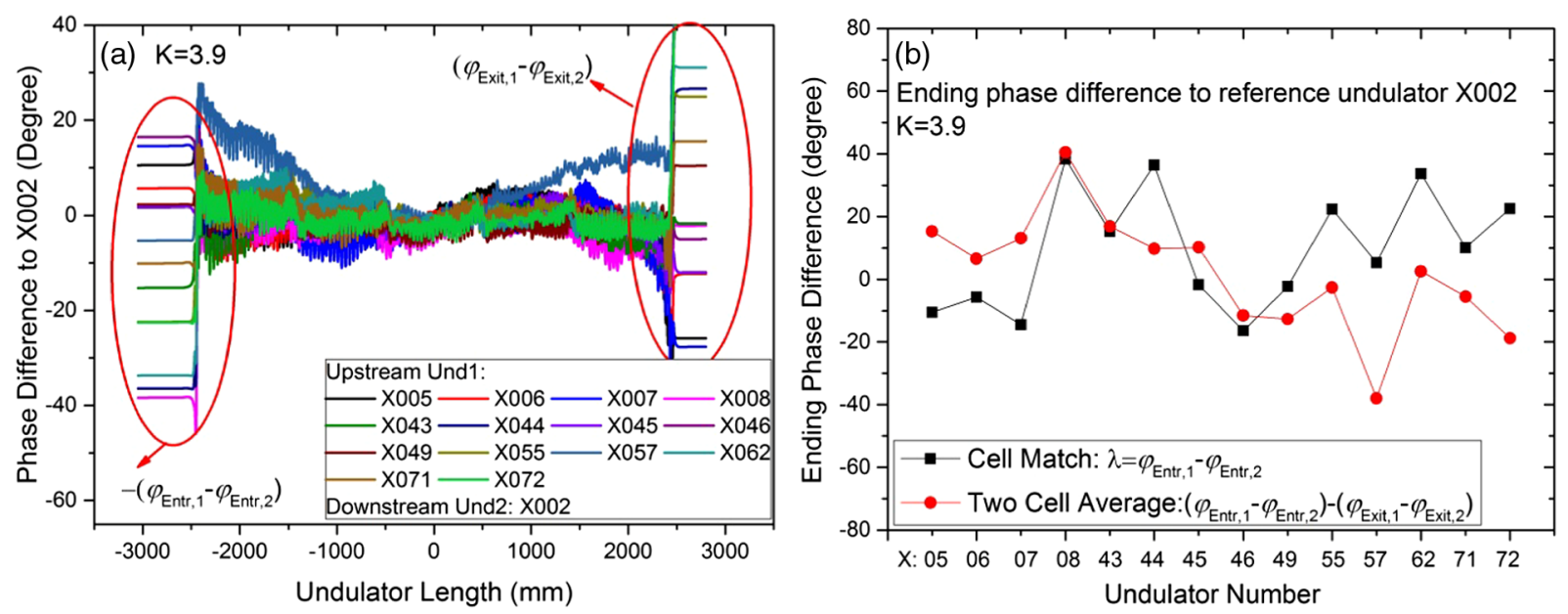

FIG. 3. The phase difference between the 14 undulators to a reference undulator. (a) The phase difference over the full length of the undulator. (b) Test of the valid conditions for the simplified matching methods.

However at first two attempts were made, which made assumptions on magnetic field properties and could simplify phase matching by avoiding the interpolation of the field maps.

1. Cell matching.-The assumption is that the phase advance over an undulator cell is given by $\varphi_{\text {Und }}+\varphi_{\mathrm{PS}}=2 n \pi$. It does not make the distinctions described in the previous section. This method only considers a single isolated undulator cell and does not consider its neighbors. It also does not distinguish phase advance in the regions of fringe field and the bulk field. Comparing these simplified criteria with Eq. (12), the condition $\varphi_{\text {entr, } 1}=\varphi_{\text {entr, } 2}$ results. It means this simplification works only when the phase advance in the entrance fringe field has, within some tolerance limits, the same value for all undulators. This assumption was assessed.

2. Two cell averaging. - The criteria is $\left(\varphi_{\mathrm{Und}, 1}+\varphi_{\mathrm{Und}, 2}\right) /$ $2+\varphi_{\mathrm{PS}}=2 n \pi$. It takes into account the contribution from both undulators upstream and downstream a phase shifter but omits the distinction between the fringe and bulk fields. In comparison with Eq. (12) the condition for this method to work isv $\varphi_{\text {entr }, 1}+\varphi_{\text {exit, } 2}=\varphi_{\text {exit, } 1}+\varphi_{\text {entr }, 2}$. It means the sum of the phase advance in the entrance region of the upstream undulator and in the exit region of the downstream undulator equals the sum of phase advance in the exit region and the entrance region from the upstream and downstream undulators respectively.

For both methods only the total phase advance over the whole undulator is required. For each undulator segment it meticulously recorded as a function of the gap. Phase matching is greatly simplified if these data can be used and the detailed calculation as described in the previous section could be avoided.

In order to test these two assumptions data from 15 representative undulator segments were selected. One, the undulator $\mathrm{U} 40-\mathrm{X} 002$ is used as the reference and the other
14 are compared to it. Interpolation of the K vs gap and phase vs gap curves was used to obtain consistent data for $\mathrm{K}=3.9$.

The calculation results are shown in Fig. 3. Figure 3(a) illustrates the difference of the optical phase between the selected 14 undulators with respect to the reference undulator X002. The origin of the phase is set in the undulator centers. Differences extend up to $\pm 40^{\circ}$. The constant regions before and after the undulator correspond to the field free regions where the phase difference stays constant. The different values reflect the differences. The phase advance in the entrance and exit region differs significantly segment to segment. Figure 3(b) visualizes the differences for the cell matching and two cell averaging. It is evident that the ending sections are quite different and differences extend up to $\pm 40^{\circ}$ and cannot be accepted. Interpolation for the fields map is unavoidable.

\section{B. Accurate matching techniques 1. Field fitting}

In order to obtain the field for a specific $K$ the interpolation of field maps measured at discrete gaps in the immediate vicinity is needed. This includes five steps.

1. Using the measured field maps as a function of gap the undulator K-parameters are calculated and a discrete function $K_{m}\left(g_{m}\right)$ is established. The suffix $m$ denotes discrete measured values. The standard measurement data include field maps made with gap steps of $0.5 \mathrm{~mm}$ steps from 10 to $22 \mathrm{~mm}$ and 10 more point up to $100 \mathrm{~mm}$. The operational gap range from 10 to $20 \mathrm{~mm}$ is thus well covered.

2. The coefficients $a_{i}$ of a polynomial $K(g)=\sum_{i=0}^{n} a_{i} g^{i}$ are fitted to the discrete set of $K_{m}\left(g_{m}\right)$. The maximum order, $n$, is typically $6 . K(g)$ is a smooth continuous function. 

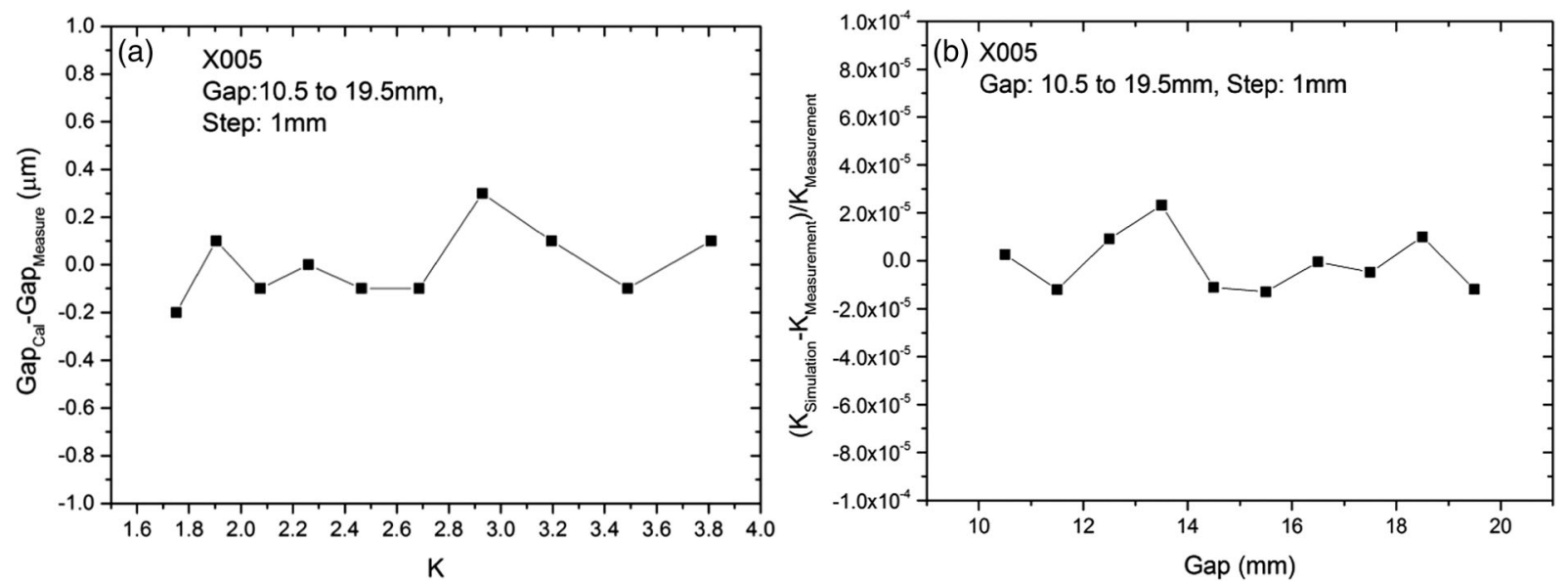

FIG. 4. Test of the interpolation using gap steps of 0.5 and $1.0 \mathrm{~mm}$. (a) Differences of the gap are well within $0.4 \mu \mathrm{m}$; (b) Differences of the K-parameter are within $\pm 2 \times 10^{-5}$.

3. The polynomial fitting is inverted so that a smooth continuous interpolation function $g(K)$ is obtained, which is used to determine the exact gap for a specific $K$. The inversion is done by a numerical point by point inversion at each sampling point performed on the polynomial coefficient $a_{i}$.

4. Field fitting: For a specific $K$ the undulator gap, $g_{0}$, is evaluated as shown in step 3 . Field maps at eight gaps, four smaller and four larger than $g_{0}$, are used for interpolation. At each point of the field map polynomial coefficients $b_{i}$ are fitted through the eight data points. At small and large gaps the number of data points needs to be adjusted accordingly.

5. Based on the coefficient $b_{i}$ the interpolated undulator field at $g_{0}$ is calculated using $f\left(g_{0}\right)=\sum_{i=0}^{n} b_{i} g_{0}^{i}$. In our case we use the sixth order polynomial fitting $n=6$.

In order to test the accuracy of the field fitting the undulator segment U40-X005 was selected. Field maps are measured over $\pm 3050 \mathrm{~mm}$ with a $0.5 \mathrm{~mm}$ step size and extend over the full length of a cell. Such a map consists of 12201 data points. The coordinate origin is in the geometric center of the undulator as was already seen in Fig. 3(a). For testing the accuracy of the interpolation a step size test was made: The gap interpolation using gaps with $1 \mathrm{~mm}$ step size was compared with results using $0.5 \mathrm{~mm}$. The difference is used to estimate the accuracy of this method.

Figure 4(a) shows the difference of the interpolated gaps for K-parameters ranging from 1.7 to 3.9 using 1 and $0.5 \mathrm{~mm}$ interpolation. The maximum difference is less than $0.4 \mu \mathrm{m}$, which is less than half the resolution of gap control and considered sufficiently accurate.

The dependence of the K-parameter on step size is shown in Fig. 4(b). Compared are two data sets each containing field measurements at 10 gap settings. One is the field based on the field fitting according to the five steps above. The original field is the scan at gaps from $10.0 \mathrm{~mm}$ up to $20 \mathrm{~mm}$. The other data set is the real measurement at gaps from 10.5 to $19.5 \mathrm{~mm}$. It is seen that the $\mathrm{K}$ difference is smaller than $2 \times 10^{-5}$. This is more than 1 order of magnitude smaller than the Pierce parameter $\rho$ and again considered accurate enough.

The difference between field interpolation and measurements already shows sufficient accuracy. In practice only $0.5 \mathrm{~mm}$ interpolation will be used, so the accuracy should be further increased. As a result interpolation using the $0.5 \mathrm{~mm}$ data provides sufficient accuracy to obtain field data for any arbitrary K-parameter.

\section{Effect on phase by compensation of ending kick}

The specifications of the European XFEL allow for residual end kicks of $\pm 0.15 \mathrm{Tmm}$ or less, which in general change with gap. Air coils, which are installed on both undulator ends, are used to compensate the residual kicks. Using Eq. (2) the change of the optical phase $\Delta \varphi$ caused by a kick $\delta_{k}$ at position $z_{0}$ is given by

$$
\begin{aligned}
\Delta \varphi(z) & =\frac{k}{2 \gamma^{2}}\left[2 \delta_{k} \int_{z_{0}}^{z} I_{1 y}\left(z^{\prime}\right) d z^{\prime}+\left(z-z_{0}\right) \delta_{k}^{2}\right] \\
& \approx \frac{k}{\gamma^{2}} \delta_{k} \int_{z_{0}}^{z} I_{1 y}\left(z^{\prime}\right) d z^{\prime} .
\end{aligned}
$$

If the location of the air coil correctors is inside of the undulator streak fields the term $\int_{z_{0}}^{z} I_{1 y}\left(z^{\prime}\right) d z^{\prime}$ is much larger than $\delta_{k}$ and it results in the approximation in Eq. (13). Therefore in Eq. (13) the main contribution to $\Delta \varphi$ is proportional to the product of the second field integral and the kick at the position $z_{0}$. Outside the undulator field only a small contribution remains. If the kick is inside the undulator field it can result in considerable phase errors. This analysis shows that the air coils need to be carefully placed in order to minimize the impact on phase matching. 

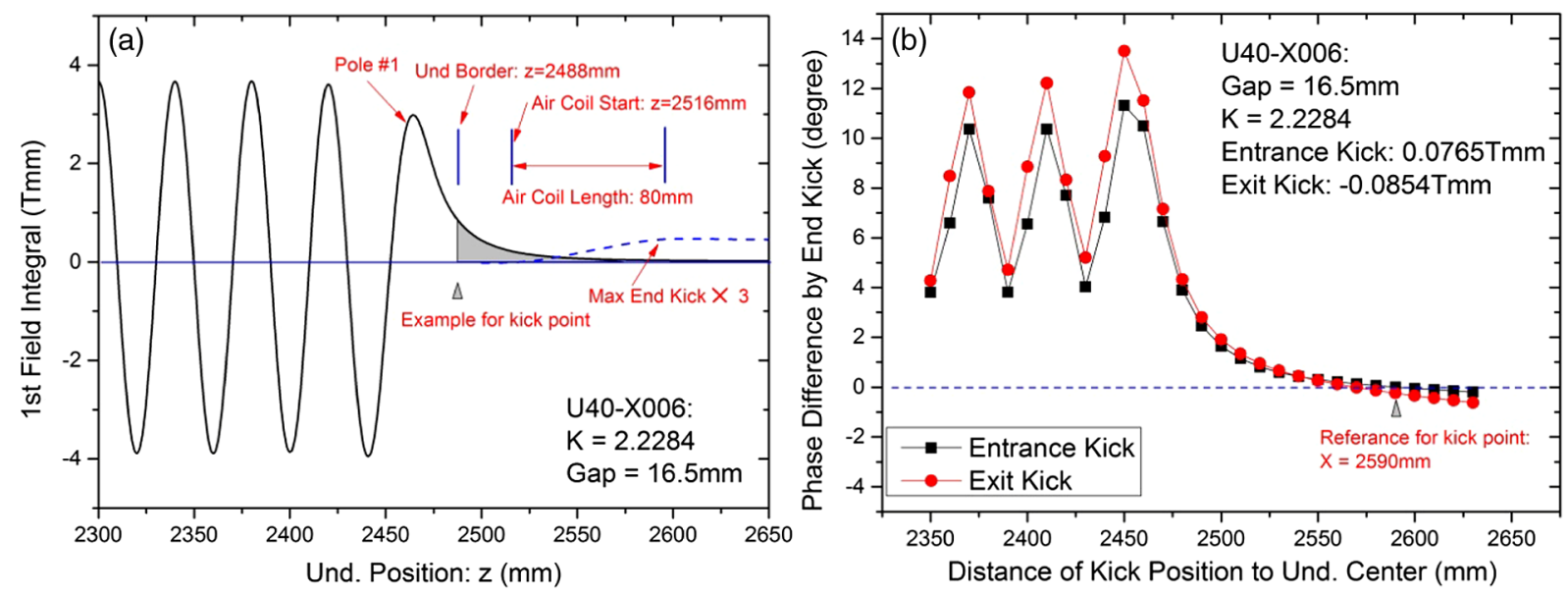

FIG. 5. Effect of the position of the air coil on optical phase. (a) First field integral of the U40-X006 undulator. The hardware border for both undulator and air coil positions are indicated. For demonstration the dashed line indicates the contribution of the air coil with full excitation of $0.45 \mathrm{Tmm}$ (b) Dependence of the phase difference on the position of the air coils at the entrance (black squares) and exit (red circles). Note the abscissa represents the distance to the center not the X-position.

Figure 5(a) illustrates the first field integral near the exit end of a representative undulator segment, the U40-X006. The gap is $16.5 \mathrm{~mm}$ and $\mathrm{K}$ value is 2.2284 . The mechanical end of the undulator at $2488 \mathrm{~mm}$ is shown. The contribution of fringe field extends to about $2570 \mathrm{~mm}$. The $80 \mathrm{~mm}$ long air coil starts at $2516 \mathrm{~mm}$ where there is some small residual nonzero contribution of the undulator field. For demonstration the blue dashed line in Fig. 5(a) represents the first field integral measured on a representative air coil located at this position with full excitation of $0.45 \mathrm{Tmm}$, which is much stronger than required. It is seen, however, that the field overlap of undulator and air coil field is very small.

Figure 5(b) illustrates quantitatively the impact on $\Delta \varphi$, which would result if the position of the air coils is changed. In order to obtain a flat trajectory throughout the undulator an entrance kick of $+0.0765 \mathrm{Tmm}$ at $-2590 \mathrm{~mm}$ and an exit kick of $-0.0854 \mathrm{Tmm}$ at $+2590 \mathrm{~mm}$ need to be applied. The reference phase is calculated with the air coils at these positions. In Fig. 5(b) the phase difference is shown, if the air coils are shifted but with unchanged strengths. The black squares show the results if the entrance coil is shifted but the exit coil is kept fixed. The red circles show the analog situation for the exit coil. Note, in order to show both ends in one plot the abscissa represents the distance to the center not the X-position since the undulator field is symmetric and extends from -3050 to $+3050 \mathrm{~mm}$. It is seen that as long as the distance of the air coils to the center is larger than $2510 \mathrm{~mm}$ the phase difference is smaller than 1 degree. With decreasing distance the phase error can increase significantly to more than 10 degrees, see also Eq. (13).

Since the air coil starts at $2516 \mathrm{~mm}$ it is concluded that the impact of the position of end kicking is $<1^{\circ}$ and therefore negligible.

\section{MATCHING RESULTS}

\section{A. General}

For demonstration of the method two undulator segments, the U40-X005 and the U40-X006 and one phase shifter, the PS073, are used. The K-parameter of the two undulators is set from 1.5 up to 3.9 with a step size of 0.2 . It corresponds to the operational range. The gap for each segment was fitted to match the desired K-parameter. Figure 6 illustrates the required gap of the two segments as a function of $\mathrm{K}$. The blue curve shows the difference. It is seen that there is an almost constant difference of about $0.15 \mathrm{~mm}$ with a very slight variation with gap. It is due to differences in the mechanics, encoder initialization and magnet structure and shows the need of individual gap

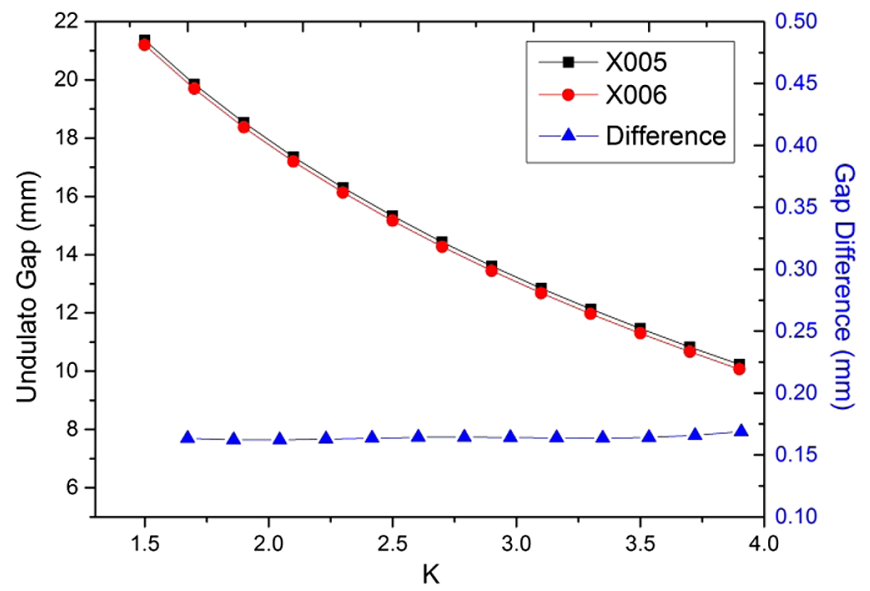

FIG. 6. Gap vs K-parameter for the U40-X005 (black squares) and U40-X006 (red circles). The difference is shown by the blue triangles. 


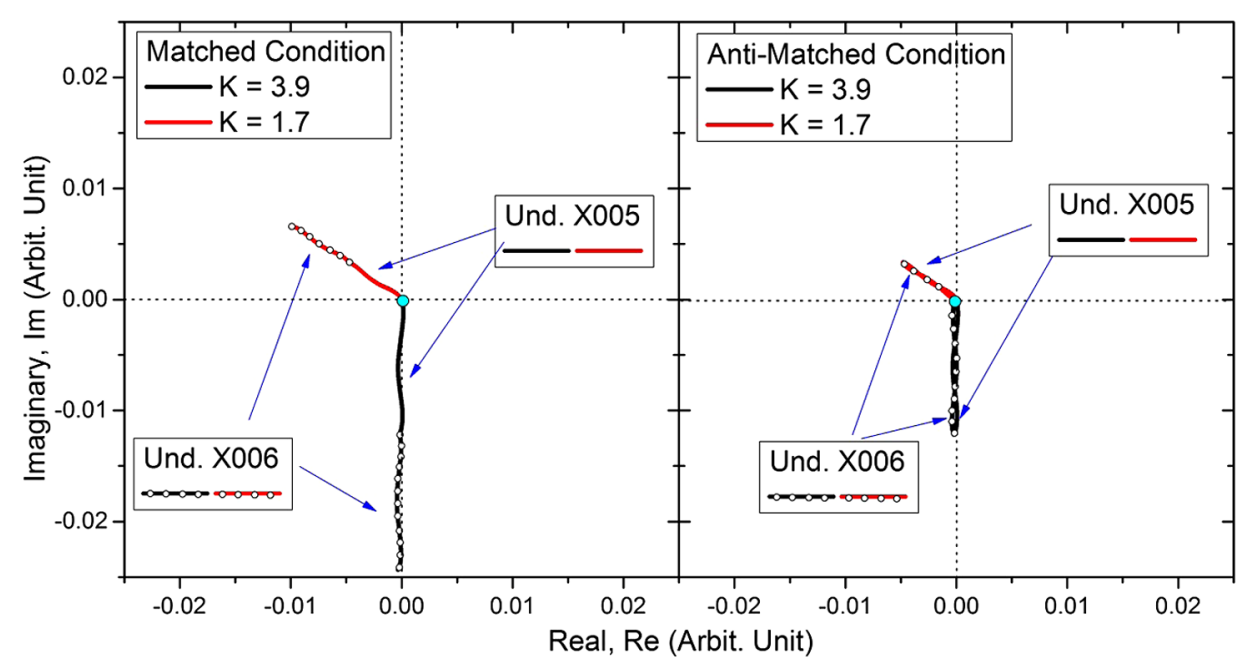

FIG. 7. Hodograph of the real and imaginary part of the radiation $A_{n}$ for two $\mathrm{K}$ values. The left plot corresponds matched phasing with $2 n \pi$ phase difference. The right plot corresponds antimatched phasing with $(2 n+1) \pi$ phase difference leading to zero amplitude in the forward direction.

adjustment. This is quite representative for other undulator segments as well.

\section{B. Phase matching using the $\boldsymbol{A}_{\boldsymbol{n}}$}

The condition for proper phase matching is defined by Eq. (7). The real and imaginary part of the normalized $A_{n}$ using Eq. (3) and the phase $\psi$ can be calculated. Figure 7 demonstrates the phase matching using the hodograph representation.

The abscissa represents the real and the y-axis the imaginary part in arbitrary units. Two cases representing the maximum and minimum $\mathrm{K}$ values, 3.9 and 1.7, are chosen for demonstration. Going along a perfect undulator the complex amplitude evolves along a straight line starting at 0 . For each $\mathrm{K}$ value, two conditions called "matched" and "antimatched" are shown. Matched fulfills the phase matching condition, Eq. (7) i.e. $2 n \pi$. For the antimatched condition the phase delay between the undulators is $(2 n+1) \pi$. Results are shown by the left and right plots, respectively. The start angle is different to various $\mathrm{K}$ value and it depends on the entrance ending field. It is seen that in the matched condition the $A_{n}$ of two of the undulators, U40-X005 and U40-X006, have the same length and point in exactly the same direction and the total amplitude of the radiation from two undulators is twice the amplitude of a single one and reaches the maximum intensity as illustrated in Fig. 2. In contrast as seen by the right plot in the antimatched condition $A_{n}$ of the two undulators have again the same length but reverse direction. Therefore the total length is zero. It should be emphasized that this applies to the forward direction only. The effect of residual field errors is seen by some small wiggles on the lines and resulting small deviations from perfect straightness.

Small differences in the ending fields as well as small individual differences in the phase shifter require strict matching of undulators and phase shifters. Figure 8 shows the required phase shifter gap as a function of the undulator K-parameter. The phase shifter can be operated on different harmonic numbers, $\nu$ [7]. Here the harmonic number means the integral times of $2 \pi$ of the phase advance in the intersection between two undulators. The harmonic number $\nu$ needs to be adjusted to the $\mathrm{K}$ range. For example if for an experiment $\mathrm{K}$ needs to be scanned from 1.5 to 3.9 harmonic $\nu \geq 14$ must be selected. At a lower $\nu$ the range cannot be covered. Figure 8 shows the results. Each curve corresponds to a specific harmonic number. It is seen that the larger the harmonic number the larger the $\mathrm{K}$ range. However at large $\mathrm{K}$ the space between the curves gets smaller and the phase gets more sensitive to the phase shifter gap.

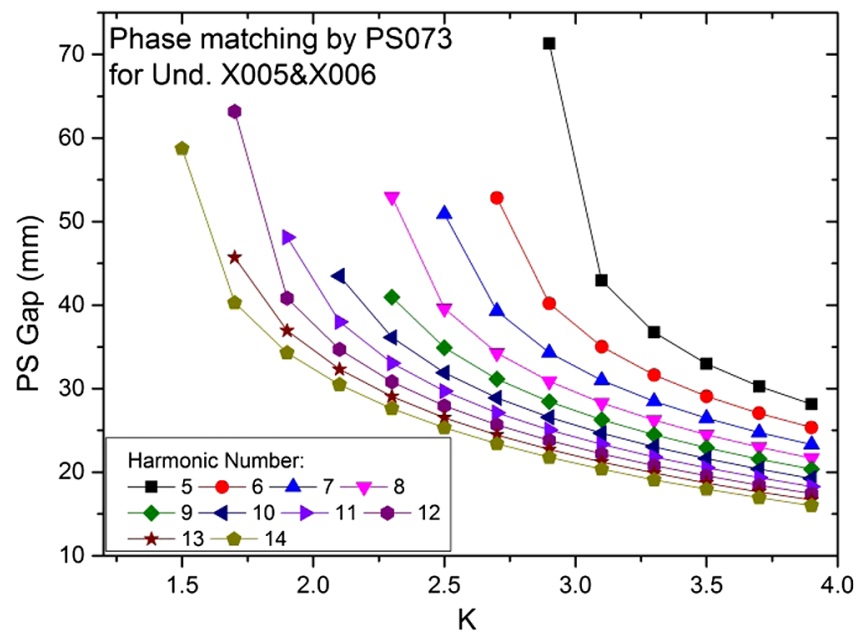

FIG. 8. The required gap of phase shifter PS073 placed between U40-X005 and U40-X006 as a function of the K-parameter. The individual curves correspond to different phase numbers. 


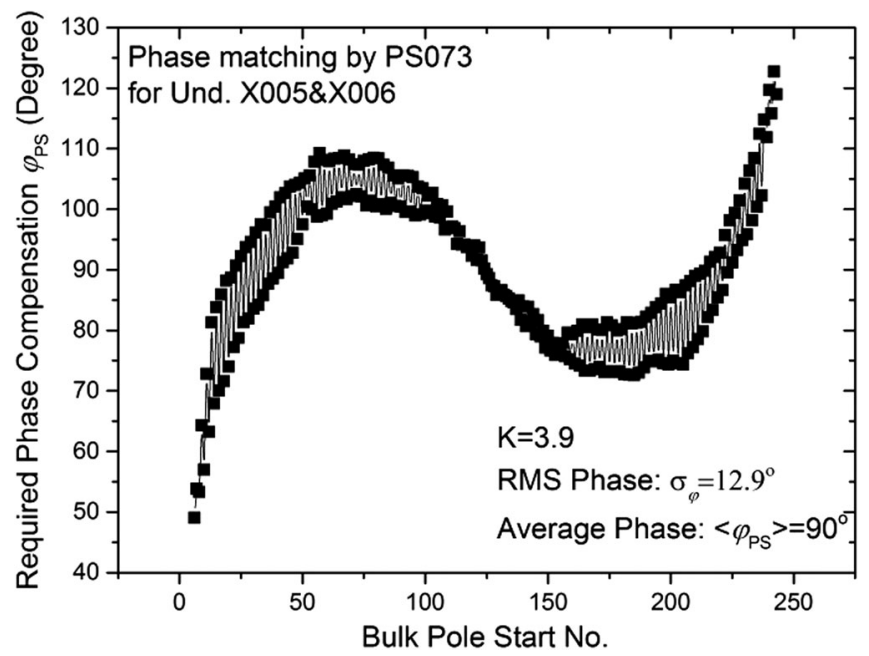

FIG. 9. Phase compensation as a function of the pole number where the bulk field starts.

\section{Phase matching using the optical phase}

Phase matching described so far is the most general treatment which is based on the complex $A_{n}$ of the two undulators calculated from their measured fields. Under the assumption that the bulk structure has zero phase error there is a simple alternative using Eq. (12). Only the end field contributes. In reality the problem arises of how to select the boundary between bulk and end fields. The applicability of this simplification was again tested using the undulators U40-X005 and U40-X006 and the phase shifter PS073.

Figure 9 shows the required phase compensation of the phase shifter as a function of the pole number where the bulk structure starts. It is seen that depending on the start the phase varies significantly from about 50 to 120 degrees with an rms value of 12.9 degrees. There is no hard criterion to select a specific pole as the start pole for the bulk field. In the bulk structure there is a systematic deviation from a $2 \pi$ phase advance per period. The explanation is that on all European XFEL undulators small parabolic girder deformations have been observed, which result from changing magnetic forces, which are well within the specifications. Therefore different extensions of the bulk field lead to different phase matching requirements [10]. Since the curve in Fig. 9 has some symmetry averaging can be used. The average of the requested phase in Fig. 9 is $90^{\circ}$ and is used to calculate the phase shifter gap. Now, using Eq. (12) phase shifter gap settings in full analogy to Fig. 8 can be calculated.

Both methods provide comparable results. Instead of reproducing curves such as in Fig. 8 a quantitative analysis is given in Fig. 10. The black curve shows the difference of the phase shifter gap setting a function of the undulator $\mathrm{K}$-parameter. It does not exceed $-7 \mu \mathrm{m}$. As shown by the blue curve the difference of the phase shifter correction is less than one degree. These differences are negligible

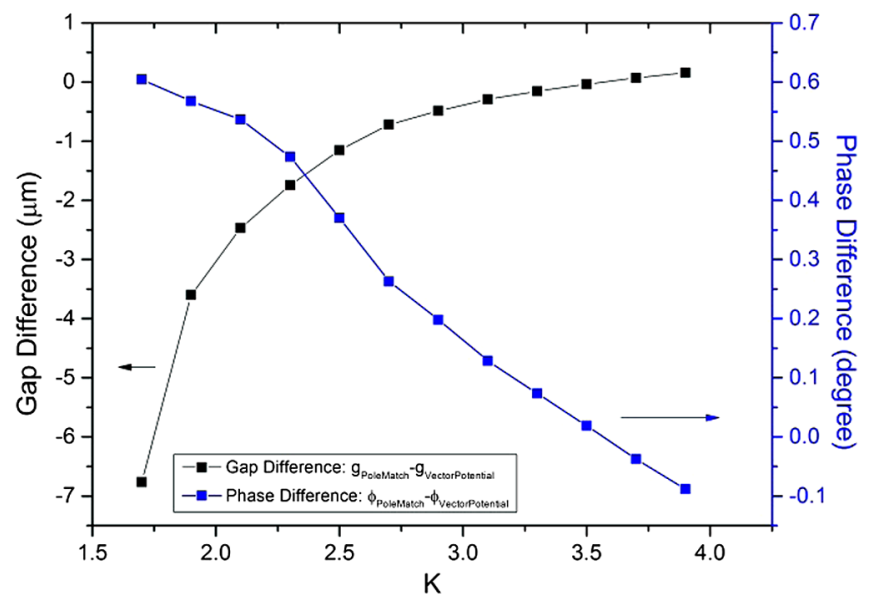

FIG. 10. Comparison between the results of two matching criteria. The black curve shows the difference of the required phase shifter gap, the blue curve the phase difference of phase shifter.

and both methods give the same phase matching and can be used for the work.

\section{EMISSION SPECTRA}

In order to further validate the phase matching method the spontaneous radiation (SR) of the two undulators and phase shifter were investigated. SR spectra are another quantitative tool to evaluate the phase matching and the overall field quality.

To start with the effect of the combination of two ideal undulators plus the phase shifter is studied analytically. The on-axis radiation of a single ideal undulator as a function of wavelength near the fundamental harmonic can be expressed by the detuning factor $\delta=\Delta \lambda_{r} / \lambda_{r}$, where $\lambda_{r}$ is the radiation wavelength and $\Delta \lambda_{r}$ is the shift of the wavelength:

$$
\begin{aligned}
A(\delta) \approx & \left(\frac{e^{2}}{8 \pi^{2} c}\right)^{\frac{1}{2}} \frac{k}{\gamma}\left[J_{0}\left(\frac{K^{2}}{1+0.5 K^{2}}\right)-J_{1}\left(\frac{K^{2}}{1+0.5 K^{2}}\right)\right] \\
& \times \frac{e^{-i 2 N \pi\left(\delta-\delta^{2}\right)}-1}{\delta} .
\end{aligned}
$$

$N$ is the undulator period number. The intensity is proportional to the square of $A$. The radiation intensity from a single undulator, $I_{\text {Single }}$, as a function of $\delta$ is proportional to

$$
I_{\text {Single }}(\delta) \propto \frac{4 \sin ^{2}(N \pi \delta)}{\delta^{2}} .
$$

Without loss of generality we consider a special condition in Eq. (12): $\varphi_{\text {exit, } 1}+\varphi_{\text {entr }, 2}=0$. The intensity of two undulators with phase delay $\varphi_{\mathrm{PS}}$ in between as the function of $\delta$ is given by 


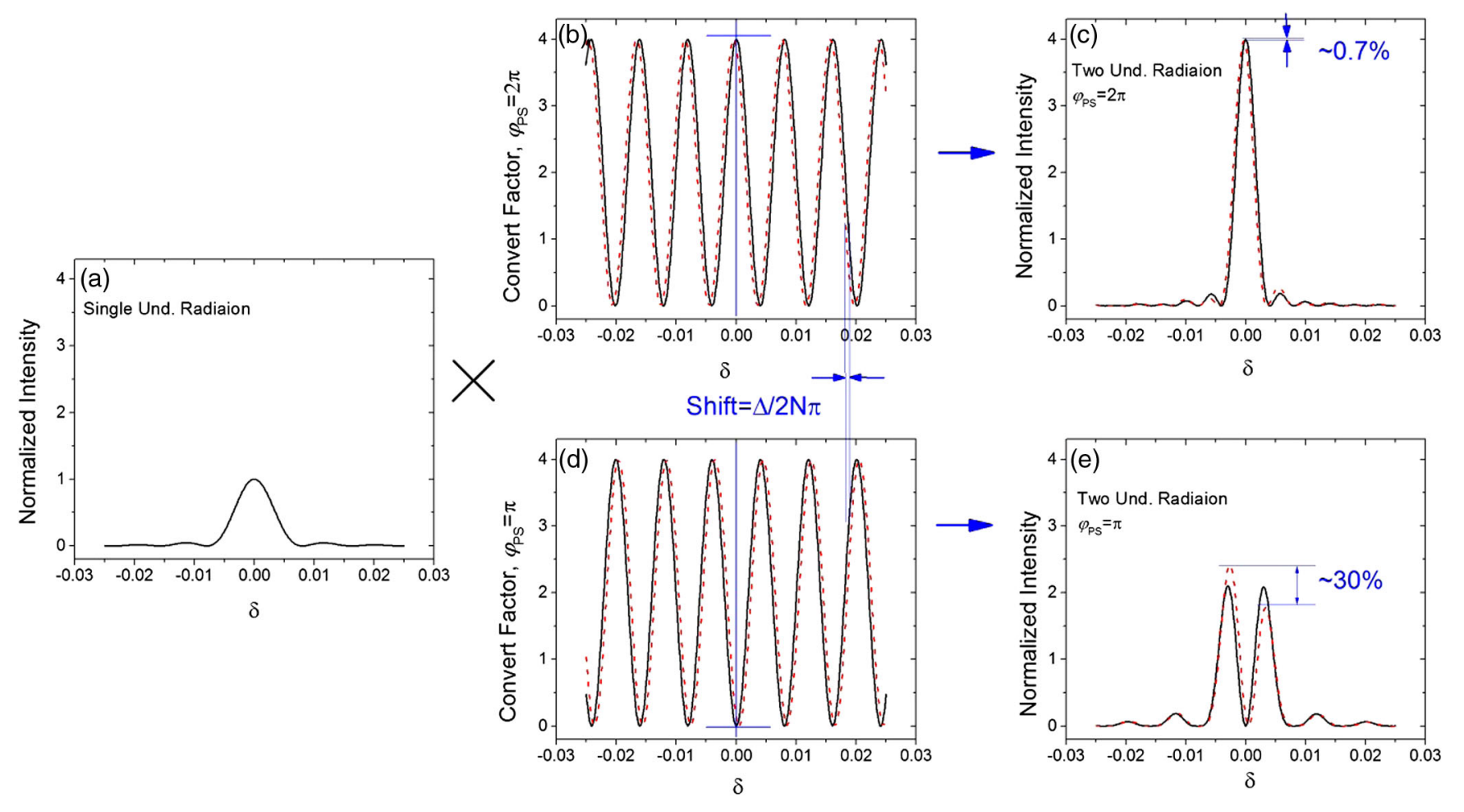

FIG. 11. The on-axis radiation spectrum from two undulators. (a) Single undulator spectrum. (b) Conversion coefficient, Eq. (17). Black curve: Phase shifter set to $\varphi_{\mathrm{PS}}=2 \pi$. Red curve: $\Delta=20$ degrees. (c) Two undulator spectrum, product of (a) and (b). There is marginal difference for $\Delta=20$ degree. (d) Conversion coefficient, Eq. (18). Black curve: Phase shifter is set to $\varphi_{\mathrm{PS}}=\pi$. (e) Two undulator spectrum, product of (a) and (d). Now there is a clear difference for $\Delta=20$ degree.

$$
\begin{aligned}
I_{\text {TwoUnd }}(\delta) & \propto\left|e^{i\left[(1-\delta) \varphi_{\mathrm{PS}}+2 N \pi \delta\right]}+1\right|^{2} I_{\text {Single }} \\
& =2\left\{1+\cos \left[(1-\delta) \varphi_{\mathrm{PS}}+2 N \pi \delta\right]\right\} I_{\text {Single }} .
\end{aligned}
$$

If there is an error $\Delta$, in the phase delay $\varphi_{\mathrm{PS}}$ in the phase matched condition $\varphi_{\mathrm{PS}}=2 n \pi$, the total intensity is proportional to

$$
I_{\text {total }}(\delta) \propto 2\{1+\cos [2 \pi(N-n) \delta]-\Delta \sin [2 \pi(N-n) \delta]\} I_{\text {und }} .
$$

For the antimatched condition with $\varphi_{\mathrm{PS}}=(2 n+1) \pi$, the total intensity is proportional to

$$
\begin{aligned}
I_{\text {total }}(\delta) \propto & 2(1-\cos \{[2(N-n)-1] \pi \delta\} \\
& +\Delta \sin \{[2(N-n)-1] \pi \delta\}) I_{\text {und }} .
\end{aligned}
$$

where $n$ is phase number and $N$ is undulator period number. Using Eqs. (16)-(18) the spectrum of two undulators with phase shifter can be calculated from the spectrum of a single one, which is expressed by Eq. (15).

Figure 11 shows spectra which were calculated using Eqs. (15)-(18). U40 undulator parameters are used with $N=125$. Figure 11(a) shows the normalized spectrum of a single undulator using Eq. (15). Figures 11(b) and 11(d) show the coefficients given by Eqs. (17) and (18) which convert the single undulator spectra to those of two undulators. Figure 11(b) represents the matched condition $\varphi_{\mathrm{PS}}=2 n \pi$ and Fig. 11(d) corresponds to the antimatched condition $\varphi_{\mathrm{PS}}=(2 n+1) \pi$. In each plot two curves are shown. The black curves correspond to zero phase shifter error, $\Delta=0$, the red dashed curves to the phase shifter detuned by 20 degrees, $\Delta=20^{\circ}$. It is seen that both conversion coefficients in Figs. 11(b) and 11(d) are sinusoidal functions of $\delta$. In the phase matched condition the coefficient reaches maximum of 4 at $\delta=0$. Therefore the on-axis intensity of two undulators is 4 times that of the single undulator, as seen in Fig. 11(c). In the antimatched condition the conversion coefficient is zero at $\delta=0$ and there is no on-axis radiation at the radiation wavelength. However, according to Eq. (18) the antimatched conversion coefficient has a maximum on axis at $\delta \approx \pm 1 / 2 N$. Therefore there are two peaks in the spectrum, as shown in Fig. 11(e).

From Eq. (15) it is seen that the single undulator intensity at $\delta=0$ is given by $I_{\text {Single }}(0)=\lim _{\delta \rightarrow 0} \frac{4 \sin ^{2}(N \pi \delta)}{\delta^{2}}=4 N^{2} \pi^{2}$ and at $\delta= \pm 1 / 2 N$ by $I_{\text {Single }}(1 / 2 N)=16 N^{2}$. The ratio is $\frac{I_{\text {und }}\left(\frac{1}{2 N}\right)}{I_{\text {und }}(0)}=\frac{4}{\pi^{2}}=0.405$. By comparing Figs. 11(c) and 11(e) the peak intensity of the antimatched condition is about half the intensity of the matched case. 
By comparison of the red and black curves in Figs. 11(b) and $11(\mathrm{~d})$ it is seen that a small phase shifter error $\Delta$ shifts the conversion coefficient by $d \delta \approx \Delta / 2 N \pi$. Since the spectrum of two undulators is the product of the conversion coefficient and the spectrum of a single undulator, the shift of the conversion coefficient has an impact on total intensity. Using Eq. (14) the change of intensity of a single undulator intensity against $\delta$ is

$$
\frac{d I_{\mathrm{und}}}{d \delta}=\frac{4\{-1+\cos [2 N \pi \delta(1-\delta)]-N \pi \delta(2 \delta-1) \sin [2 N \pi \delta(1-\delta)]\}}{\delta^{3}} .
$$

Accordingly $\lim _{\delta \rightarrow 0} \frac{d I_{\text {und }}}{d \delta}=8 N^{2} \pi^{2}$ and $\lim _{\delta \rightarrow 1 / 2 N} \frac{d I_{\text {und }}}{d \delta} \approx$ $64 N^{3} \cos ^{2}(\pi / 4 N)$ with the ratio $8 N / \pi^{2}$. For an U40 with $N=125$ it results in about 100 . The intensity changes much faster at $\delta=1 / 2 N$ than at $\delta=0$. It is seen explicitly in Fig. 11(a): When $\delta=0$, the radiation intensity is in the peak region and therefore the slope is zero. In contrast at

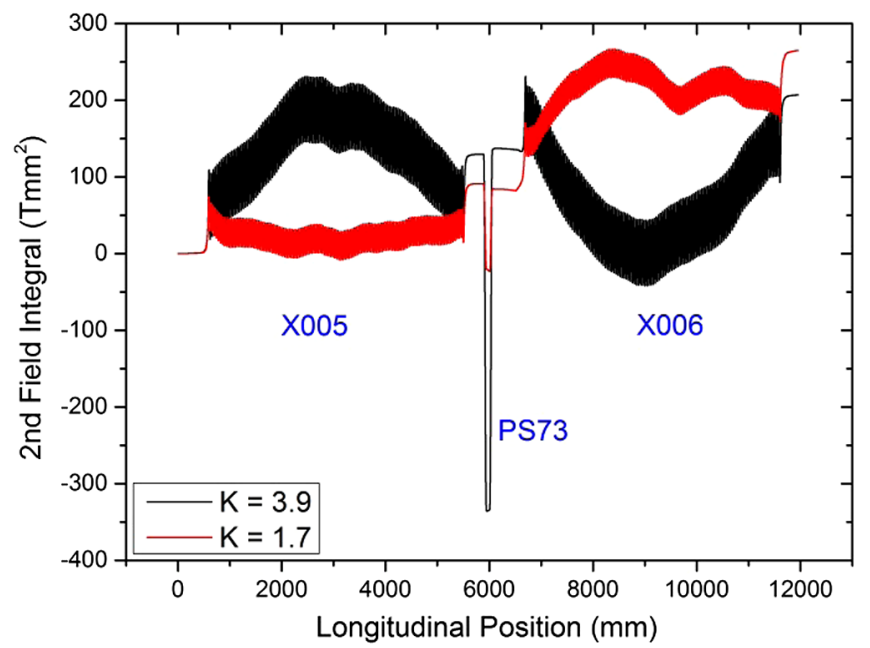

FIG. 12. Second field integrals of the two undulators U40X005 and U40-X006 in combination with the phase shifter PS073. Black $\mathrm{K}=3.9$; red $\mathrm{K}=1.7$.

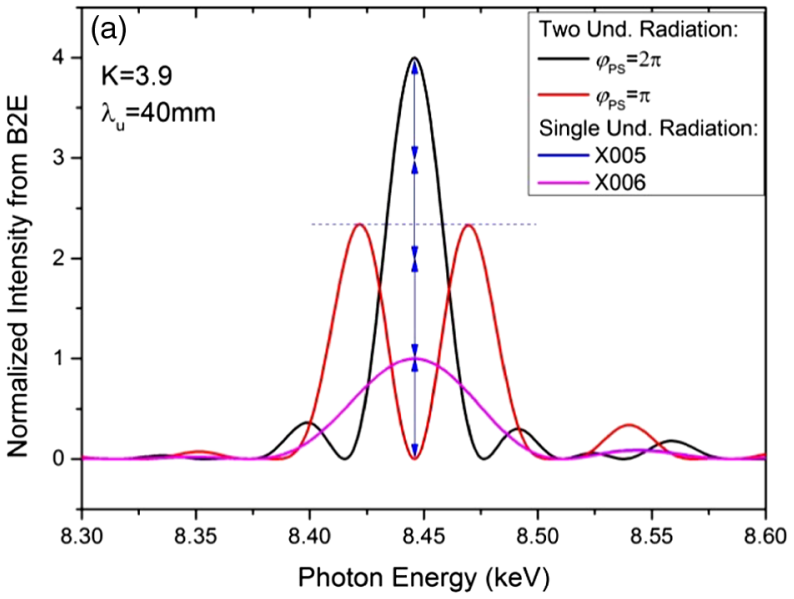

$\delta=\frac{1}{2 N}=0.004$, the intensity changes with a steep slope. The difference is important to check the tuning. For the matched condition shown in Fig. 11(c) by the black and red curves the peak intensities change only about $0.7 \%$, which is a small change. However, as mentioned above, the red curve presents a phase shifter error by 20 degrees. So the spectrum for the matched condition is not sensitive to detect a phase shifter phase error of this magnitude or less. On the contrary Fig. 11(d) shows the intensity change with the same error of 20 degrees in the antimatched condition, $\varphi_{\mathrm{PS}}=(n+1) \pi$. It is seen that the double peaks in the antimatched spectrum are much more sensitive to phase errors. The difference is about $30 \%$.

For a full simulation of high accuracy field maps measured on the U40-X005, the U40-X006 and the phase shifter PS073 were taken to calculate the radiation spectra with the SR simulation code B2E [18]. Two different K-parameters, 1.7 and 3.9, were selected and field maps were obtained by the interpolation method described in Sec. III B 1.

To begin with Fig. 12 shows the second field integrals for both undulators and the phase shifter in between. The black curve shows the $\mathrm{K}=3.9$ and the red curve the $\mathrm{K}=1.7$ results. It is seen that the two undulators and phase shifter are properly tuned with zero entrance and exit kicks. The data shown in Fig. 12 are quite representative for the XFEL undulators.

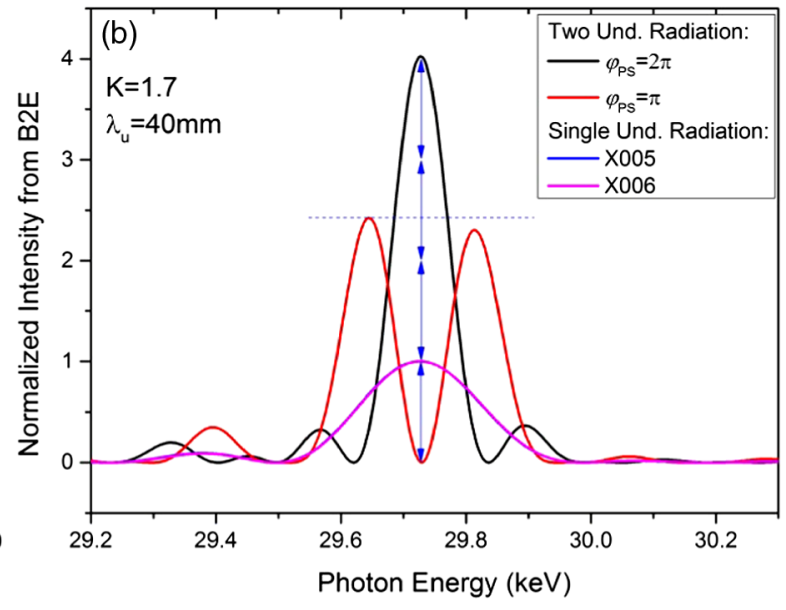

FIG. 13. On-axis spectrum calculated with B2E from measured magnet data of the undulators U40-X005 and U40-X006 and the phase shifter PS073. (a) $\mathrm{K}=3.9$; (b) $\mathrm{K}=1.7$; black curves correspond matched phase $\varphi_{\mathrm{PS}}=2 \pi$ and red curves to the antimatched phase, $\varphi_{\mathrm{PS}}=\pi$. The same relative scale applies to all curves. Single undulator spectra coincide within linewidths. Only the spectrum of the $\mathrm{U} 40-\mathrm{X} 006$ is shown, that of the U40-X005 is obscured. 


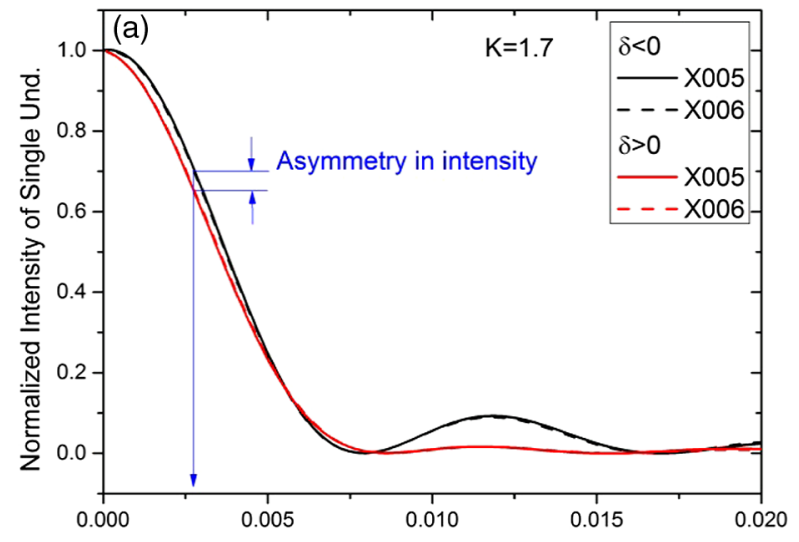

$|\delta|$

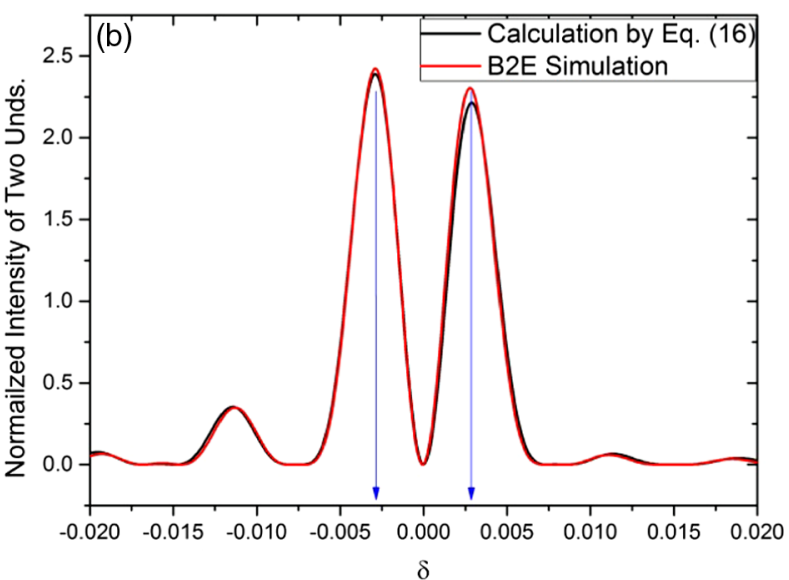

$\delta$

FIG. 14. (a) Symmetry test of single undulator spectra. Black curves $\delta<0$; red curves $\delta>0$. There is again very good agreement between the two undulators. The asymmetry in the spectrum is clearly seen on both. (b) Double undulator spectra for the antimatched phase, $\varphi_{\mathrm{PS}}=\pi$. Black curve: Combination of single undulator spectrum and Eq. (16). Red curve: Full B2E simulation.

The SR spectrum of this configuration calculated with B2E is shown in Fig. 13. The first harmonic for $K=3.9$ and $\mathrm{K}=1.7$ is shown in Figs. 13(a) and 13(b), respectively. Spectra for the matched and antimatched condition are shown by the black and red curves, respectively. The single undulator spectra for U40-X005 and U40-X006 coincide within linewidth. Therefore only the last one can be displayed.

For $\mathrm{K}=3.9$ in Fig. 13(a) the single undulator spectra of X005 and X006 agree each other very well. This indicates that in both undulators the determination of the $\mathrm{K}$-parameter and that the interpolation scheme works well within an estimated accuracy better than $1 \times 10^{-5}$. There is a lot of similarity with the analytic treatment shown in Fig. 11. The intensity of the phase matched double undulator spectra is 4 times that of the single.

For $\mathrm{K}=1.7$ shown in Fig. 13(b) the double peak for the antimatched phase shows a slight asymmetry, while the others are quite similar. One explanation could be a slight phase detuning by an estimated $2.6^{\circ}$ as seen in Fig. 11(e). Such a detuning could be neglected. However, another reason was found by analyzing the symmetry of the line shape of the single undulator spectra for $K=1.7$. Figure 14(a) shows the negative side of the single undulator line transformed to the positive side. It is seen that there is an asymmetry: For positive $\delta$ data are slightly lower than for negative values. From Eq. (18) it is seen that for the antimatched phase this asymmetry would lead to an according asymmetry of the double undulator spectrum. Figure 14(b) shows this comparison: Single undulator spectrum combined with Eq. (16), black line and full B2E calculation, red. In general there is very good agreement within linewidth. An according analysis of the line shape for $\mathrm{K}=3.9$ did not show an asymmetric line shape as seen in Fig. 14(a). So the dominant contribution to the asymmetry seen in Fig. 13(b) comes from the line shape.

\section{SUMMARY AND CONCLUSIONS}

In this paper the theoretical basis for the proper matching the optical phase of different tunable undulator segments with the help of phase shifters as found in large distributed undulator systems for SASE FELs are worked out.

The following aspects were treated and investigated.

1. It was found that due to unavoidable manufacturing tolerances there are always slight differences in the K-parameters for different undulators at the same gap, which exceed the limits given by the Pierce parameter, $\frac{\Delta K}{K}<\rho$. The gap of each undulator needs to be adjusted individually to match the required K-parameter.

2. Each undulator needs to be characterized by extensive magnetic measurements made at different gaps. These measurements are the basis for proper phase matching. For EXFEL field maps are measured at various gaps in $0.5 \mathrm{~mm}$ steps.

3. In order to obtain field maps for a specific K-parameter these field maps need to be interpolated. The applied interpolation scheme was demonstrated to achieve an accuracy of $\frac{\Delta K}{K}$ better than $2 \times 10^{-5}$ using these data.

4. Using these interpolated field maps the radiation complex $A_{n}$ is used to determine the proper phase shifter settings between two undulators for any K-parameter in the operational range.

5. Air coil correctors need to be placed outside the undulator fringe field so that they do not deteriorate the phase matching.

6. Two representative U40 undulators of the European XFEL were chosen. The following observations are made: (a) Although the fields of two undulators look quite similar 
the phase advance in the end field sections between the two ends of the same undulator as well as between different undulators are quite different and cannot be neglected. The phase advance per period in the bulk field differs from the ideal $2 \pi$ due to slight gap dependent girder deformation. Consequently individual treatment of each undulator segment is needed to achieve sufficient accuracy for overall phase matching. (b) Therefore the fields of both undulators need to be taken into account for the phase matching. (c) As demonstrated for a representative phase shifter the exact settings of the phase shifter gap as a function of the undulator K-parameter can be calculated for different phase numbers. Contiguous tuning over the whole operational gap range of a XFEL $\mathrm{U} 40$ from $\mathrm{K}=1.7$ to 3.9 requires a minimum phase number of 14 .

7. The phase matching was cross-checked with SR calculations using B2E and measured field data from two undulators and one phase shifter. The results showed: (a) The intensity in the forward direction is not sensitive to phase matching errors if the phase matching is $2 n \pi$. This will be the usual operation mode. An error of $20^{\circ}$ only results in a reduction of intensity of less than $1 \%$. (b) The forward intensity of the two undulators is 4 times that of the individual undulators. This is a good indication for the field quality. (c) The antimatched phase condition with $(2 n+1) \pi$ is much more sensitive to phase matching errors. Although this mode cannot be used for lasing it provides a tool for precise phasing. It was observed that the single undulator line shapes are slightly asymmetric, which leads to the same effect as a small phase mismatch. However, this effect was found quite small.

In summary the analysis of the data showed that their quality is sufficient and proper phase matching of the XFEL undulators requires to apply the methods described in this paper.

The methods described in this paper are exclusively based on magnetic data, which cannot be applied in situ. A similar study has been made in Refs. [19,20]. For later refinement and cross-checking of K-parameter and phase shifter settings optical diagnostic tools as already discussed in [21] are under development for the European XFEL $[22,23]$.

[1] A VUV free electron laser at the TESLA test facility, TESLA-FEL Conceptual Design Report No. 95-03, 1995.

[2] B. Faatz et al., Flash II: Perspectives and challenges, Nucl. Instrum. Methods Phys. Res., Sect. A 635, S2 (2011).

[3] Linac Coherent Light Source Conceptual Design Report No. SLAC-R-593 UC-414, 2002, available under http://www.slac.stanford.edu/cgi-wrap/getdoc/slac-r-593.pdf http://www-ssrl.slac.stanford.edu/lcls/cdr/.

[4] Linac Coherent Light Source II Conceptual Design Report No. SLAC-R-978; I-060-003-000-00, 2011, available under http://www.slac.stanford.edu/pubs/ slacreports/reports19/slac-r-978.pdf.

[5] M. Altarelli et al., The European X-ray Free-electron Laser, Technical Design Report, 2006, http://www.xfel .eu/dokumente/technical_documents.

[6] The FERMI project at Elletra, Conceptual Design Report No. ST/F-TN-07/12, 2007, available under https://www .elettra.trieste.it/lightsources/fermi/fermi-machine/fermicdr .html.

[7] H. Lu, Y. Li, and J. Pflueger, The permanent magnet phase shifter for the European X-ray Free Electron Laser, Nucl. Instrum. Methods Phys. Res., Sect. A 605, 399 (2009).

[8] J. Pflueger et al., Status of the undulator systems for the European X-ray Free Electron Laser, in Proceeding of FEL2013, New York, NY USA (2013), TUPSO60.

[9] Y. Li, S. Abeghyan, K. Berndgen, M. Bagha-Shanjani, G. Deron, U. Englisch, S. Karabekyab, B. Ketenoglu, M. Knoll, F. Wolff-Fabris, M. Viehweger, M. Yakopov, and J. Pflueger, Magnetic measurement techniques for the large-scale production of undulator segments for the European XFEL, Synchrotron Radiat. News 28, 23 (2015).

[10] Y. Li, B. Ketenoglu, and J. Pflueger, Girder deformation related phase errors on the undulators for the European X-Ray Free Electron Laser, Phys. Rev. ST Accel. Beams, 18, 060704 (2015).

[11] Y. Yang, Y. Li, H. Lu, F. Wolff-Fabris, and J. Pflueger, Shimming strategy for the phase shifters used in the European XFEL, Proceedings of the FEL2013, New York, NY, USA (2013), TUPSO42.

[12] Y. Li and J. Pflueger, High accuracy shimming techniques ofr the phase shifters of the European XFEL, in Proceeding of FEL2014, Basel, Switzerland (2014), MOP007.

[13] Y. Li and J. Pflueger, Tuning method for phase shifters with very low first field integral errors for the European X-ray Free Electron Laser, Phys. Rev. ST Accel. Beams 18, 030703 (2015).

[14] U. Englisch, T. Wei, P. Li, Y. Li, F. Wolff-Fabris, and J. Pflueger (to be published).

[15] J. D. Jackson, Classical Electrodynamics (John Wiley \& Sons Inc., New York, 1962), Chap. 14.

[16] R. P. Walker, Interference effects in undulator and wiggler radiation sources, Nucl. Instrum. Methods Phys. Res., Sect. A 335, 328 (1993).

[17] S. Krinsky, M. L. Perlman, and R. E. Watson, in Handbook of Synchrotron Radiation, edited by E. E. Koch, D. E. Eastmans, Y. Farge et al. (North-Holland Publishing Company, Amsterdam, 1983), Vol. 1, Chap. 2.

[18] B2E is a software dedicated to the simulation of the radiation produced by a relativistic single electron traveling through an arbitrary magnetic field. It is available at http://www.esrf.eu/Accelerators/Groups/ InsertionDevices/Software/B2e.

[19] E. Gluskin, N. A Vinokurov, G. Decker, R. J Dejus, P. Emma, P. Ilinski, E. R Moog, H.-D. Nuhn, and I. B Vasserman, Optimization of the design for the LCLS undulator line, Nucl. Instrum. Methods Phys. Res., Sect. A 475, 323 (2001). 
[20] B. Diviacco, R. Bracco, D. Millo, and M. Musardo, Phase shifters for the FERMI@Elettra undulators, in Proceedings of the 2nd International Particle Accelerator Conference, San Sebastián, Spain (EPS-AG, Spain, 2011), pp. 3278-3280.

[21] M. Tischer, P. Illinski, U. Hahn, J. Pflüger, and H. SchulteSchrepping, Photon diagnostik for the x-ray FELs at TESLA, Nucl. Instrum. Methods Phys. Res., Sect. A 483, 418 (2002).
[22] J. Grünert, J. Buck, C. Ozkan, W. Freund, and S. Molodtsov, X-ray photon diagnostics devices for the European XFEL, Proc. SPIE 8504, 85040R (2012).

[23] W. Freund, The undulator commissioning spectrometer for the European XFEL, European XFEL Technical Note 1-57, 2014 [XFEL.EU TN-2014-00101], available at https://bib-pubdb1.desy.de/record/ 170410/files/DESY-2014-03060.pdf. 